Disclosure of Interest: None declared

DOI: 10.1136/annrheumdis-2017-eular.4294

\section{THU0545 COMPARISON OF THE CLINICAL AND LABORATORY FEATURES AT ONSET BETWEEN MULTICENTRIC CASTLEMAN'S DISEASE AND ADULT-ONSET STILL'S DISEASE}

A. González García ${ }^{1}$, W.A. Sifuentes Giraldo ${ }^{2}$, J.L. Morell Hita ${ }^{1}$, J.L. Patier de la Peña ${ }^{2} \cdot{ }^{1}$ Rheumatology; ${ }^{2}$ Internal Medicine, Ramon y Cajal University Hospital, Madrid, Spain

Background: Multicentric Castleman's disease (MCD) is a disorder characterized by polyclonal proliferation of B lymphocytes that is frequently associated with autoimmune manifestations and connective tissue diseases. MCD presents high levels of IL-6 and systemic symptoms such as fever, arthralgia, hepatosplenomegaly and serositis, so it is recommended to include MCD in the differential diagnosis of adult-onset Still's disease (AOSD). However, there are no studies comparing both groups of patients.

Objectives: To compare the clinical and laboratory features at onset between patients with MCD and AOSD seen in a Madrid tertiary care hospital.

Methods: We performed a retrospective observational study in patients with diagnosis MCD and AOSD attended our center between January 1989 and December 2015. The variables included demographics, clinical manifestations, laboratory tests and Yamaguchi's criteria.

Results: A total of 34 patients were included, 17 with MCD and 17 with AOSD. The comparison of the characteristics of both groups is presented in the table. There were no differences in age, uration of disease (MCD 158,6 days and AOSD 250,5 days, $p=0,3919$ ), diagnostic delay (MCD 18,4 and AOSD 52,2, $\mathrm{p}=0,2711$ ), arthritis, myalagias, pleuritis or macrophagic activation syndrome, but persistent fever, rash, arthralgia, pharyngitis and pericarditis were significantly more frequent in ESA, whereas male gender, human immunodeficiency virus (HIV) and/or human herpes virus 8 (HHV8) infection, hepatosplenomegaly and lymphadenopathy were significantly higher in MCD. In the laboratory test results, leukocytosis and hypertransaminasemia were significantly higher in AOSD. 52.9\% of patients with MCD met 5 or more Yamaguchi's criteria for AOSD.

\begin{tabular}{lccc}
\hline & $\begin{array}{c}\text { Multicentric Castleman's } \\
\text { disease }(\mathrm{n}=17)\end{array}$ & $\begin{array}{c}\text { Adult-onset Still's } \\
\text { disease }(\mathrm{n}=17)\end{array}$ & $\mathrm{p}$-value \\
\hline Male:female ratio & $2.4: 1$ & $0.4: 1$ & 0.0163 \\
Age at diagnosis (mean $\pm \mathrm{SD})$ & $46.4 \pm 13.1$ & $37.5 \pm 13.5$ & 0.0598 \\
HIV infection & $35.2 \%$ & $0 \%$ & 0.0154 \\
HHV8 infection & $35.2 \%$ & $0 \%$ & 0.0154 \\
Persistent fever & $47.1 \%$ & $94.1 \%$ & 0.0078 \\
Rash & $29.4 \%$ & $64.7 \%$ & 0.0392 \\
Arthralgias & $35,3 \%$ & $88.2 \%$ & 0.0014 \\
Arthritis & $0 \%$ & $17.6 \%$ & 0.0696 \\
Pharyngitis & $0 \%$ & $41.2 \%$ & 0.0029 \\
Hepatosplenomegaly & $47.1 \%$ & $5.9 \%$ & 0.0065 \\
Pericarditis & $0 \%$ & $23.5 \%$ & 0.0332 \\
Pleuritis & $17.6 \%$ & $11.7 \%$ & 0.6217 \\
Lymphadenopathy & $100 \%$ & $17.6 \%$ & 0.0001 \\
Macrophage activation syndrome & $11.7 \%$ & $17.6 \%$ & 0.6282 \\
Leucocytes $>10000 / \mathrm{mm}^{3}$ & $35.2 \%$ & $76.5 \%$ & 0.0156 \\
Increased transaminases & $0 \%$ & $58.8 \%$ & 0.0002 \\
Erythrosedimentation rate (mm/Hg) & 52 & 77.5 & 0.1104 \\
C-reactive protein (md/dl) & 75.1 & 109.5 & 0.3699 \\
Ferritin (ng/ml) & 240 & 1926 & 0.1234 \\
\hline
\end{tabular}

Conclusions: Patients with MCD may present common systemic manifestations and laboratory abnormalities at onset with AOSD and up to $50 \%$ of them may fulfill Yamaguchi's criteria for this disease, so MCD should be taken into account in its differential diagnosis.

Disclosure of Interest: None declared

DOI: 10.1136/annrheumdis-2017-eular.6228

\section{THU0546 IMPACT OF BONE LOCALISATION ON THE PROGNOSTIC OF LANGERHANS CELL HISTIOCYTOSIS: A MONOCENTRIC RETROSPECTIVE STUDY}

J.-G. Letarouilly ${ }^{1}$, N. Segaud ${ }^{1}$, B. Wallaert ${ }^{2}$, P.-Y. Hatron ${ }^{3}$, R.-M. Flipo ${ }^{1}$ ${ }^{1}$ Rhumatologie, CHRU Lille Hôpital Roger Salengro; ${ }^{2}$ Pneumologie, CHRU Lille Hôpital Calmette; ${ }^{3}$ Médecine interne, CHRU Lille Hôpital Huriez, Lille, France

Background: Langerhans cell histiocytosis $(\mathrm{LCH})$ is a rare condition, and mostly affects children. Bone is the most commonly involved organ, with bone lesions in $50 \%$ of patients. In a recent work, Arico et al. described that the probability of survival in children suffering from a multisystemic LCH with risk organ involvement was reduced if patient did not have any bony lesion [1]. There is no such a study in adult patients.

Objectives: The objective of the study was to know the bone impact on the prognosis of adult $\mathrm{LCH}$

Methods: A retrospective monocentric study was performed using data from the patients hospitalized for a LCH at Centre Hospitalier Régional Universitaire de Lille, a university hospital between 2001 and 2015. All patients with LCH and at an age of 18 years or older were included. Patients were excluded if they did not receive any osteoarticular imaging (radiography, scintigraphy, PET, MRI).

Results: Our study initially included 70 patients had LCH. After screening 54 patients met the inclusion criteria: 31 had bone localization (BLG) and 23 none (NBLG). The two groups showed differences. The lesion leading to the diagnosis was mostly osteoarticular (18 patients) in BLG and pulmonary (18 patients) in NBLG. The BLG presented more multisystemic form than the NBLG (20 vs 2, $p<0.0001)$. Both groups were equivalent for comorbidities $(p=0,206$ for cancer and $p=0,756$ for cardiovascular disease), number of smokers and age at diagnosis (36.8+/- 14.15 years vs $36.7+/-12.6$ years, $\mathrm{p}=0.96$ and 25 vs $22, \mathrm{p}=0.06$ ). Treatment was required for 14 patients in BLG and for 2 in NBLG. In BLG, 8 patients were treated medically, mostly by corticosteroid therapy and chemotherapy (7), one patient underwent radiotherapy. 6 patients were treated by surgery. In NBLG, 2 patients were treated by corticosteroid therapy. There was no surgery in NBLG (table 1). 3 patients died in BLG, 2 directly related to $L C H$ and one from postoperative infectious complications. One patient died in NBLG from hematologic pathology (chronic myelomonocytic leukemia). The time between diagnosis and death was one, six and 22 months in BLG, 36 months in NBLG. 7 patients presented relapses in BLG. 5 had been treated by medical treatment, one surgically and one had no previous treatment. The time of relapse was six, $8,12,14,16,52$ and 72 months. 4 presented new bone lesions, 1 pulmonary lesions, 1 dermatologic lesions and 1 neurologic lesion (table 1). There was no relapse in NBLG. Unfortunately, there was a lack of statistical power to conclude about the bone impact on the prognosis.

Conclusions: Patients with bone location and those with no bone location are two different phenotypes of adult LCH. Patients with bone location are more proned to have a multisystemic relapsing disease. A multicentric study with a larger number patients is needed to bring more robust data to answer about bone impact on the prognosis of adult $\mathrm{LCH}$.

\section{References:}

[1] Aricò M, Astigarraga I, Braier J, et al. Lack of bone lesions at diagnosis is associated with inferior outcome in multisystem langerhans cell histiocytosis of childhood. Br J Haematol 2015;169:241-8.

Disclosure of Interest: None declared

DOI: 10.1136/annrheumdis-2017-eular.6790

\section{THU0547 CHARACTERIZATION OF A GROUP OF 12 PATIENTS WITH MEVALONATE KINASE DEFICIENCY: SYMPTOMS AND TREATMENT WITH IL- 1 INHIBITORS}

A. Kozlova $^{1}$, V. Burlakov ${ }^{1}$, N. Kuzmenko ${ }^{1}$, S. Zimin ${ }^{2}$, T. Varlamova ${ }^{1}$, O. Barabanova ${ }^{2}$, E. Raikina ${ }^{1}$, A. Shcherbina ${ }^{1}{ }^{1}{ }^{1} /$ mmunology, Federal State Budgetary Institution "National Scientific and Practical Center of Pediatric Hematology, Oncology and Immunology named after Dmitry Rogachev" of the Ministry of Healthcare of the Russian Federation; ${ }^{2}$ Immunology, Children's Clinical Hospital N9, Moscow, Russian Federation

Background: Mevalonate kinase deficiency (MKD) is a rare autosomal recessive autoinflammatory disease caused by mutations in MVK gene. MKD patients typically have an early onset of symptoms including recurrent episodes of high fever, abdominal pain, diarrhea and vomiting, arthralgia and lymphadenopathy (AIDAI criteria for HIDS). However not all patients have typical symptoms at the time of onset. MKD treatment remains an unsolved problem, since none of the modalities previously used for MKD treatment are fully effective in the disease control.

Methods: We conducted a retrospective analysis of clinical features of twelve patients ( 6 females, 6 males) with genetically confirmed MKD. Nine patients received therapy with inhibitors of IL-1 (Anakinra and/or Canakinumab). One of the patients died from amyloidosis and macrophage activation syndrome (MAS) prior to treatment initiation, her diagnosis was verified post mortem.

Results: Ten patients had manifested within the first 6 months of life, one - at the age of 1.5 years, one - at three years of age,. During the course of the disease all patients had periodic fever and peripheral lymphadenopathy (mainly cervical group), as well as abdominal pain, nausea/vomiting. Five patients had diarrhea, sometimes with blood, one patient suffered from severe constipation. Rash was seen in eight patients, myalgia, artralgia were observed only in six. Oral ulcers were noted in seven children. Three patients had neurological involvement, one patient had it as the main symptom. One patient had periorbital edema and hyperemia during attacks, which to our knowledge, have not been reported previously in MKD. One patient developed amyloidosis and MAS before IL1 inhibitor treatment initiation, which led to her death. In patients receiving anti-IL-1 therapy AIDAl index decreased from $58.3 \pm 11,2$ before to $1,5 \pm 1,4$ after 6 month of therapy $(\mathrm{p}=0.003)$.

Conclusions: MKD symptoms can be variable and sometimes atypical, which requires physician's awareness. In our cohort of MKD patients anti IL-1 therapy was highly effective.

Disclosure of Interest: None declared

DOI: 10.1136/annrheumdis-2017-eular.2238 